DE

M E D I C I N A

T R O P I C A L

$\mathrm{DE}$

S ÃO PAULO

JOURNAL OF THE SÃO PAULO INSTITUTE OF TROPICAL MEDICINE
${ }^{1}$ Hospital Banting, Pharmacy Department, Banting, Selangor, Malaysia

${ }^{2}$ Hospital Banting, Orthopedic Department, Banting, Selangor, Malaysia

Correspondence to: Lim Ming Chiang Hospital Banting, Pharmacy Department, Jalan Sultan Alam Shah, 42700, Banting, Selangor, Malaysia

Tel: +603 31871333, +60169038540

E-mail: ashton5230@gmail.com

Received: 12 July 2020

Accepted: 16 November 2020

\section{Pediatric osteomyelitis due to rare tropical multi-drug resistance (MDR) organisms: a treatment quandary}

\author{
Chan Jing $\mathrm{Er}^{\circledR 1}$, Wong Kin Chun ${ }^{\circledR 2}$, Lim Ming Chiang ${ }^{\circledR}$, Mohd Naim bin \\ Mohd Nasir $^{\circledR 2} 2$
}

\section{ABSTRACT}

Osteomyelits due to concurrent multi-drug resistance organisms is difficult to treat for any surgeon and infectious disease physician. An eleven-year-old boy presenting with an open fracture of the left radius and ulna after a fall in a stagnant wet field. Despite prophylactic antibiotics and surgical intervention, the open wound was infected, and Chromobacterium violaceum as well as Klebsiella pneumoniae were isolated. He was treated with six weeks of parenteral cefepime and amikacin and was discharged upon clinical improvement. Unfortunately, chronic osteomyelitis set in with persistent sinus drainage. He then underwent a second procedure for debridement of the wound and Burkholderia pseudomallei was isolated. Parenteral antibiotic therapy was initiated progressing with a marked improvement. However, the long course of antibiotics had exhausted the patient and his family, leading to a premature interruption of the parenteral antibiotic. Despite the suboptimal antibiotic course, there were no signs of relapsed osteomyelitis during subsequent review. The timely surgical intervention with appropriate sampling for subsequent microorganism isolation guided the suitability of the treatment line.

KEYWORDS: Osteomyelitis. Multi-drug resistance (MDR) organisms. Burkholderia pseudomallei. Chromobacterium violaceum.

\section{BACKGROUND}

Chromobacterium violaceum is a gram-negative, facultative anaerobic coccobacillus widely distributed in the soil and waterfalls of tropical rainforest in Malaysia ${ }^{1,2}$. Humans are exposed to $C$. violaceum through multiple routes including direct intake of contaminated water, exposure of wounds to contaminated sources, or through contaminated medical equipment. Reported cases of human infections are rare and normally associated with a high fatality rate, due to the ability of the disease to rapidly deteriorate within $72 \mathrm{~h}$, progressing to severe sepsis with metastatic abscesses in multiple organs, then to septic shock and ultimately to death ${ }^{3}$. Even with the timely initiation of the antibiotic treatment, relapses may occur if inadequate antibiotics are used or discontinued prematurely or, in the presence of abscesses, due to viable microorganisms that still remain in the abscess cavity even after the end of the antibiotics therapy ${ }^{4}$.

Burkholderia pseudomallei is a gram-negative bacterium that causes melioidosis. It is especially prevalent in South East Asia countries including Malaysia, Thailand, Vietnam and Singapore ${ }^{5}$. The bacterium is intrinsically multi-drug resistant, but prolonged treatment with appropriate combinations of antibiotics is normally sufficient to eradicate the infection. Patients with diabetes mellitus, chronic 
renal disease and immunocompromised conditions are at higher risk for melioidosis ${ }^{6}$. The overall fatality rate among patients diagnosed with melioidosis remains significantly high despite the use of recommended standard antibacterial agents for therapy, highlighting the importance of early identification and intervention. Thus, we present a case that is, to our knowledge, the first documented case reporting on an open fracture infection due to rare tropical microorganisms involving C. violaceum and B. pseudomallei.

\section{CASE REPORT}

An 11-year-old Malay boy was presented to the Emergency Department (ED) with a chief complaint of pain on his left upper limb due to an alleged fall from a monkey bar at school onto accumulated stagnant water. At ED, he was diagnosed with an open fracture in the left radius and ulna. Immediate irrigation and immobilization of the fractured forearm were performed. His vital signs were stable. The patient was then given a dose of intravenous (IV) cefuroxime $750 \mathrm{mg}$, paracetamol syrup $390 \mathrm{mg}$ and intramuscular (IM) tetanus toxoid $0.5 \mathrm{mg}$.

The patient had no history of chronic illnesses or allergies to any food or medications. The growth and development of the child were uneventful. The wound debridement was carried out and an intramedullary K-wire was inserted in both, radius and ulna, on the next day after the patient's admission. The radiograph of the post wound debridement and intramedullary $\mathrm{K}$-wire insertion in the left radius and ulna showed acceptable reduction and fixation of the bones (Figure 1). The patient began antibiotic therapy with IV cefuroxime $650 \mathrm{mg}$ three times daily in the ward. Despite the successful surgery, the patient started to have multiple fever episodes on day 5 of admission and other parameters suggestive of infection. The laboratory findings during admission were reported in Table 1. Upon wound inspection, there was pouring of pus over the debrided

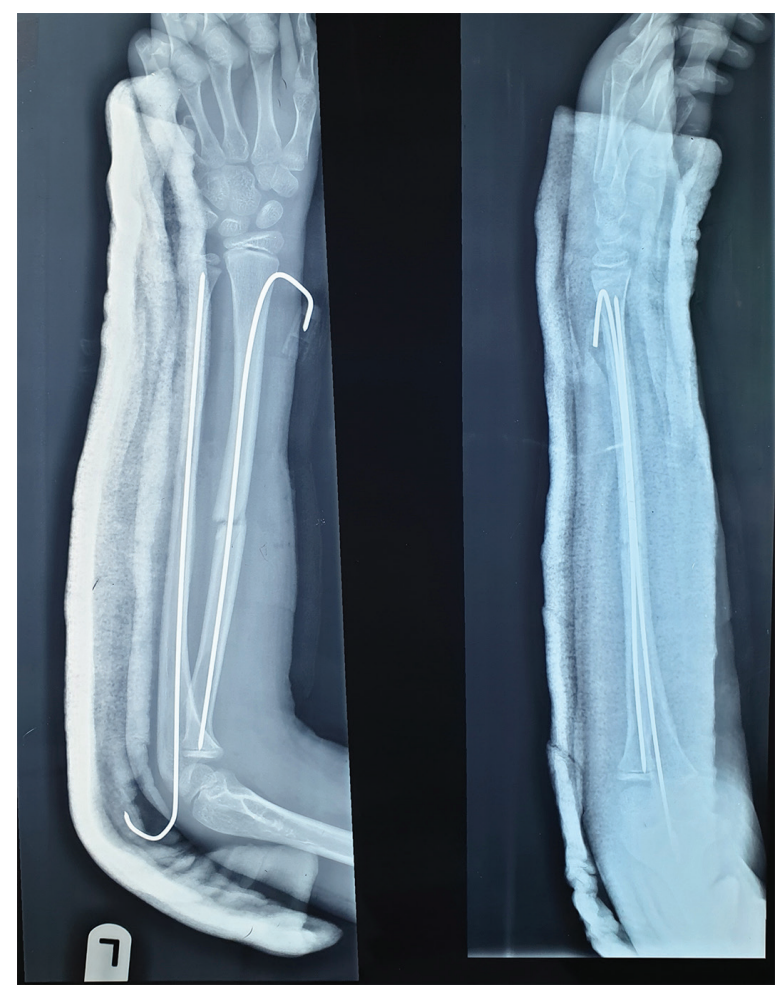

Figure 1 - Radiograph of post wound debridement and insertion of intramedullary K-Wire in the left radius and ulna with acceptable reduction and fixation.

wound. The wound debridement was repeated in view of the persistent sero-purulent discharge on the seventh day, with ulna intra-medullary K-wire removed and the radius K-wire retained. A tissue biopsy and a samples of pus were sent to culture and sensitivity (C\&S) tests were repeated during the wound debridement. The results revealed the presence of $K$. pneumoniae and $C$. violaceum infection. C\&S showed that $K$. pneumoniae was sensitive to cefuroxime, gentamicin and amikacin, while $C$. violaceum was sensitive to ciprofloxacin, gentamicin and cefepime, but resistant to ceftazidime and piperacillin/ tazobactam. The primary team decided to change to a definitive treatment with IV ciprofloxacin $260 \mathrm{mg}$ twice daily based on the C\&S

Table 1 - Laboratory findings of the patient during the first admission.

\begin{tabular}{lccccccc}
\hline \multicolumn{2}{c}{ Laboratory $\begin{array}{c}\text { parameters and reference } \\
\text { ranges }\end{array}$} & \multicolumn{7}{c}{ Date } \\
\cline { 2 - 7 } & $4-11 \times 10^{9} / \mathrm{L}$ & 7.2 & 5.8 & 10.7 & 12.0 & 8.8 & 5.9 \\
\hline TWCC & $11.5-16.5 \mathrm{~g} / 100 \mathrm{~mL}$ & 12.7 & 7.0 & 11.8 & 10.1 & 11.8 & 10.9 \\
$\mathrm{Hb}$ & $150-400 \times 10^{9} / \mathrm{L}$ & 317 & 168 & 330 & 631 & 603 & 309 \\
Platelet & $35-50 \mathrm{~g} / \mathrm{L}$ & - & - & - & 35 & 39.4 & 40.5 \\
Albumin & $53-141 \mathrm{IU} / \mathrm{L}$ & - & - & - & 180 & 226 & 243 \\
$\mathrm{ALP}$ & $<10 \mathrm{mg} / \mathrm{L}$ & - & - & - & 75.4 & 15.5 & 4.2 \\
$\mathrm{CRP}$ & & &
\end{tabular}

TWCC = Total white cell count; $\mathrm{Hb}=$ Hemoglobin; ALP = Alkaline phosphatase; CRP = C-reactive protein 
results, meantime awaiting the decision of the infectious disease physician.

An abdominal ultrasound was carried out after the identification of $C$. violaceum to rule out the dissemination of the infection. The ultrasound revealed a segment $\mathrm{V}$ liver lesion measuring $1.3 \mathrm{~cm} \times 0.8 \mathrm{~cm} \times 1.3 \mathrm{~cm}$ corresponding to an early liver abscess, an enlarged liver expansion, measuring $15.9 \mathrm{~cm}$. The patient's liver function test (LFT) has also shown a rise of alkaline phosphatase (ALP). After the pediatric infectious disease team evaluation, the antibiotic was changed to IV cefepime $1.4 \mathrm{~g}$ three times daily and IV amikacin $420 \mathrm{mg}$ once daily in view of the risk of irreversible bone and joint damage due to the use of fluoroquinolones in a young boy. Wound healing and epithelialization were observed with no discharges during the hospital stay. No adverse effects were reported with the use of the current regimen. Repeated swab $C \& S$ and blood $\mathrm{C} \& \mathrm{~S}$ showed no growth of any other microorganisms. IV amikacin was continued for two weeks, while IV cefepime was administered for up to six weeks. A repeated ultrasound prior to discharge showed resolution of the previous liver abscess. The patient was discharged with close monitoring and follow-up at the outpatient clinic. Unfortunately, one month later, a chronic osteomyelitis of ulna set in with persistent sinus drainage at the wound site. The radiograph showing osteomyelitis of the left ulna with presence of periosteal reaction and sequestration is shown in Figure 2.

The patient was readmitted to the ward and underwent another wound debridement and sequestrectomy of the left

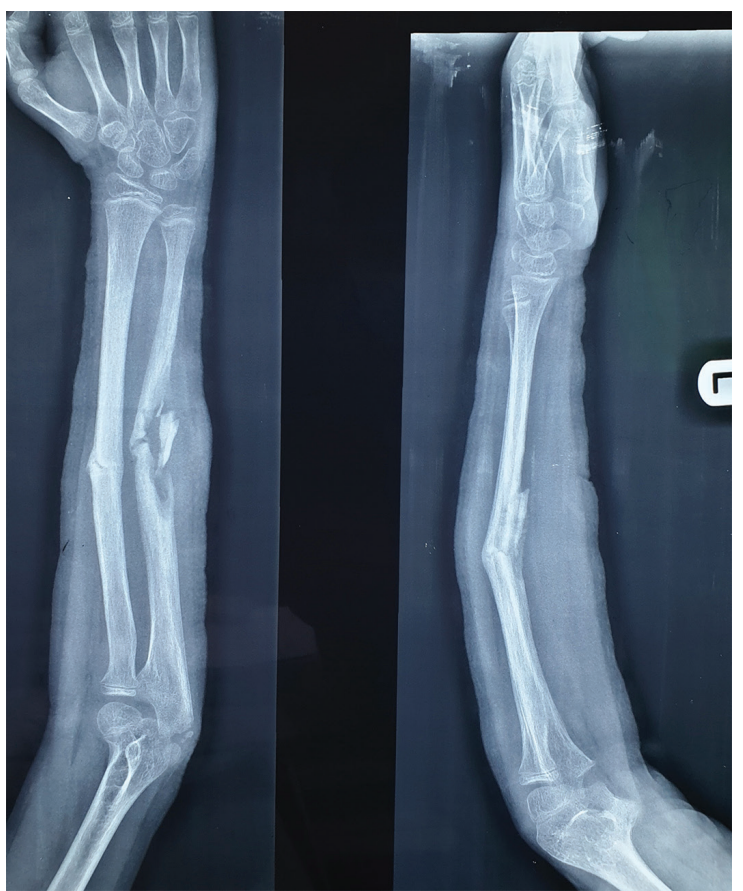

Figures 2 - Radiograph of osteomyelitis of left ulna with presence of periosteal reaction and sequestration. ulna. The bone $\mathrm{C} \& \mathrm{~S}$ taken during this surgical procedure reported the isolation of $B$. pseudomalle $i$ and methicillin sensitive Staphylococcus aureus; B. pseudomallei was sensitive to ceftazidime, imipenem and doxycycline. Thus, IV ceftazidime $1.8 \mathrm{~g}$ every four times daily was initiated parenterally, with a target duration of six weeks. The abdominal ultrasound and the echocardiogram were requested to rule out the dissemination of the infection and of abscesses or vegetations. The patient was screened for immunological abnormalities and for glucose-6-phosphate dehydrogenase (G6PD) deficiency and he was found to be normal. A marked improvement was shown along the course of treatment. However, the long course of antibiotic therapy had exhausted the patient and his family, leading to premature interruption of the parenteral antibiotic administration on day 22. Amoxicillin/ clavulanate tablets $625 \mathrm{mg}$ three times daily were given as the discharge medication to complete a total of 24 weeks. Despite the suboptimal parenteral antibiotic course, his wound and hand function recovered remarkably well and there were no signs of relapsing osteomyelitis upon the subsequent follow-up.

\section{DISCUSSION}

Our patient presented with a simple, uncomplicated open fracture, which underwent a successful surgical intervention and an empirical coverage with a parenteral antibiotic. However, the poor wound healing raised our concern and another wound debridement was performed with $C \& S$ showing the presence of a rare tropical microorganism, $C$. violaceum. We decided to initially use a single agent (ciprofloxacin) due to the lower rates of resistance found in the literature ${ }^{7}$. However, the therapy switched to a combination of IV cefepime and amikacin due to its proven synergistic effect ${ }^{8}$ after consulting an infectious disease physician and the worry of irreversible joint and bone damage secondary to the use of ciprofloxacin ${ }^{9}$. We decided for an extended six weeks duration of parenteral antibiotics, as previous cases had shown that a premature interruption of antibiotics may result in the relapsing of infection and rapid deterioration ${ }^{1,10}$. The patient responded well to the treatments and completely eradicated bacteria with no significant adverse events.

C. violaceum could cause dissemination of infection in susceptible individuals leading to liver and lung abscesses and fatal sepsis. The ALP of our patient was elevated. However, this could be normal for children and adolescents due to the development of bones ${ }^{11}$. Therefore, an abdominal ultrasound was requested to rule out the presence of liver abscesses. The ultrasound report suggested the possibility of an early abscess, thus, a close monitoring of LFT was 
carried out throughout the hospitalization and the patient's clinical condition was stable, with no signs and symptoms of acute liver injury.

The patient's condition was further complicated when we isolated B. pseudomallei from a second wound debridement samples. B. pseudomalle $i$ is an endemic tropical organism in Malaysia, usually isolated in wounds contaminated with soil or muddy water ${ }^{12,13}$. After the previous combination cefepime and amikacin, B. pseudomallei and S. aureus should have been eradicated, as they were both susceptible to cefepime. Thus, we considered the new findings as a sequential infection and decided for an initial plan of IV ceftazidime for six weeks and continuation of the oral antibiotic for a total of 24 weeks, as indicated in local guidelines ${ }^{5,14}$. Nevertheless, the patient's family decided to discharge the boy at their own risk, even after being counselled on the potential risks of the early discontinuation of antibiotics. Despite the suboptimal therapy of only three weeks of the parenteral antibiotic, the patient progressed to full recovery.

In a nutshell, timely intervention, wound debridement, repeated wound exploration and appropriate sampling for microorganism isolation could guide the suitability of treatment. Clinicians should be aware of the potentially fatal infections and the need for aggressive antimicrobial combinations after the adequate understanding of the available laboratory and diagnostic data. This is to prevent chronic infections due to opportunistic pathogens leading to increase morbidity and functional loss.

\section{ACKNOWLEDGMENTS}

We wish to acknowledge the help and advice provided by all the supporting staff especially the Department of Pediatrics and Department of Pathology during patient's admission.

\section{CONFLICT OF INTERESTS}

No reported conflict of interests.

\section{FUNDING}

The work was not funded by any agency thus the publication of this study was not dependent on the sponsor's approval or censorship of the manuscript.

\section{REFERENCES}

1. Yang $\mathrm{CH}$, Li YH. Chromobacterium violaceum infection: a clinical review of an important but neglected infection. J Chin Med Assoc. 2011;74:435-41.
2. Tan WS, Yunos NY, Tan PW, Mohamad NI, Adrian TG, Yin WF, et al. Freshwater-borne bacteria isolated from a Malaysian rainforest waterfall exhibiting quorum sensing properties. Sensors. 2014;14:10527-37.

3. Sharmin S, Jahan AA, Kamal SM, Sarker P. Fatal infection caused by Chromobacterium violaceum: a case report from a tertiary care hospital in Bangladesh. Case Rep Infect Dis. 2019;2019:6219295.

4. Richard KR, Lovvorn JJ, Oliver SE, Ross SA, Benner KW, Kong MY. Chromobacterium violaceum sepsis: rethinking conventional therapy to improve outcome. Am J Case Rep. 2015; 16:740-4.

5. Samy RP, Stiles BG, Sethi G, Lim LH. Melioidosis: clinical impact and public health threat in the tropics. PLoS Negl Trop Dis. 2017;11:e004738.

6. Hassan MR, Pani SP, Peng NP, Voralu K, Vijayalakshmi N, Mehanderkar R, et al. Incidence, risk factors and clinical epidemiology of melioidosis: a complex socio-ecological emerging infectious disease in the Alor Setar region of Kedah, Malaysia. BMC Infect Dis. 2010;10:302.

7. Lin YD, Majumdar SS, Hennessy J, Baird RW. The spectrum of Chromobacterium violaceum infections from a single geographic location. Am J Trop Med Hyg. 2016;94:710-6.

8. Mimoz O, Jacolot A, Leotard S, Hidri N, Samii K, Nordmann P, et al. Efficacies of cefepime, ceftazidime, and imipenem alone or in combination with amikacin in rats with experimental pneumonia due to ceftazidime-susceptible or-resistant Enterobacter cloacae strains. Antimicrob Agents Chemother. 1998;42:3304-8.

9. Adefurin A, Sammons H, Jacqz-Aigrain E, Choonara I. Ciprofloxacin safety in paediatrics: a systematic review. Arch Dis Child. 2011;96:874-80.

10. Ke L, An KP, Heng S, Riley M, Sona S, Moore CE, et al. Paediatric Chromobacterium violaceum in Cambodia: the first documented case. Trop Doct. 2012;42:178-9.

11. Otero JL, González-Peralta RP, Andres JM, Jolley CD, Novak DA, Haafiz A. Elevated alkaline phosphatase in children: an algorithm to determine when a "wait and see" approach is optimal. Clin Med Insights Pediatr. 2011;5:15-8.

12. Cheng AC, Currie BJ, Dance DA, Funnell SG, Limmathurotsakul D, Simpson A, et al. Clinical definitions of melioidosis. Am J Trop Med Hyg. 2013;88:411-3.

13. Nathan S, Chieng S, Kingsley PV, Mohan A, Podin Y, Ooi MH, et al. Melioidosis in Malaysia: incidence, clinical challenges, and advances in understanding pathogenesis. Trop Med Infect Dis. 2018;3:25.

14. Malaysia. Ministry of Health. National antimicrobial guideline 2019. Selangor: Ministry of Health; 2019. [cited 2020 Nov 20]. Available from: https://www.pharmacy.gov.my/v2/ sites/default/files/document-upload/national-antimicrobialguideline-2019-full-version-3rd-edition.pdf 